\title{
EXCITATION ENERGIES OF BARIUM OXIDE BANDS MEASURED IN FLAMES
}

\author{
J. van Der Hurk, TJ. Hollander and C. Th. J. Alkemade \\ Fysisch Laboratorium, Universiteit Utrecht, Sorbonnelaan 4, The Netherlands
}

(Received 14 June 1974)

\begin{abstract}
Experiments are described that yield additional information about the excitation energy of visible barium oxide bands appearing in flames. Excitation energy differences are derived directly from the ratios of thermal band intensities as a function of temperature and agree with the value calculated from the known (relative) energy-level diagram. Absolute excitation energies are derived from the temperature dependence of the ratio of band-to-line intensity under thermal equilibrium conditions and under the assumption of a most probable value for the dissociation energy of $\mathrm{BaO}$. Flames with temperatures ranging from 1911 to $2886 \mathrm{~K}$ were used. The excitation energy values found can be reconciled with the assumption that the lower ${ }^{1} \Sigma$ state of the optical transitions is the electronic ground state only if there are low-lying electronic levels that contribute at least a factor of 6 to the electronic partition function.
\end{abstract}

\section{INTRODUCTION}

THE ELECTRONIC level diagram of the alkaline-earth monoxides, and especially the possible existence of low-lying triplet states, have been subjects of controversial discussions for a long time now. ${ }^{(1)}$ The available experimental results and theoretical calculations show a disappointing lack of agreement.

HULDT and LAGERQvisT ${ }^{(2,3)}$ measured the temperature dependence of the intensity of some strong calcium, strontium, and barium bands in acetylene-air and hydrogen-oxygen flames, and derived excitation energies therefrom. Their calcium and strontium results implied that none of the bands considered combined with the electronic ground state, whereas their results for barium were consistent with the assumption of a $A^{1} \Sigma \rightarrow X^{1} \Sigma$ transition of $\mathrm{BaO}$. Their interpretation of the calcium and strontium results, however, was based on the assumption that the bands were emitted by the monoxides, which has later been shown to be false. ${ }^{(4)}$ VerTs and Gurvich ${ }^{(5)}$ measured the logarithm of the dissociation constants of some group II metal oxides as a function of the reciprocal temperature in flames with temperatures ranging from 2300 to $3200 \mathrm{~K}$. They concluded from their results that the electronic ground state of the alkaline-earth oxides is ${ }^{\mathrm{I}} \boldsymbol{\Sigma}$. BulEwiCZ and SUGDeN, ${ }^{(6)}$ however, studying the intensity of the green MgO band system in flames as a function of temperature and flame gas composition, concluded that the lower ${ }^{1} \Sigma$ state involved in the band transitions must lie at least $0.47 \mathrm{eV}$ above the ground state. DrowarT and coworkers $^{(7)}$ assumed in their mass-spectrometric measurements that the lower ${ }^{1} \Sigma$ state occurring in the band transitions was the ground state of the alkaline-earth oxides. The expected ${ }^{3} \Sigma$ and ${ }^{3} \Pi$ states, that are formed from the atoms in their ground states, were found not to lie close to the $X^{1} \Sigma$ state but rather to the $A^{1} \Sigma$ state; perturbations were observed only in the latter state in the rotational analysis of $\mathrm{BaO}$ band spectra. KLEMPERER and coworkers ${ }^{(8,9)}$ studied the microwave spectrum of $\mathrm{BaO}$ with the molecular-beam electric-resonance technique. Their values of the rotational constants agreed very well with those of the lower ${ }^{1} \Sigma$ state observed by LAGERQVIST and coworkers. ${ }^{(10)}$ They did not find any indication of electronic states with angular momenta $\Omega \geqslant 0$ below the lower ${ }^{1} \Sigma$ state and, therefore, they considered the lower ${ }^{1} \Sigma$ state of Lagerqvist to be the ground state of $\mathrm{BaO}$. They did not exclude the possible presence of low-lying excited states but 
regarded their existence as unlikely. The same authors, in a similar study of the electronic structure of SrO, ${ }^{(1)}$ showed with the aid of a magnetic deflection experiment that the lower ${ }^{1} \Sigma$ state involved in the optical bands is almost certainly the ground state of SrO. The evidence for possible triplet ground states or low-lying states in $\mathrm{SrO}$ and the other alkaline-earth oxides was reviewed and the conclusion was drawn that a triplet ground state has not been established for alkaline-earth oxides.

HOLLANDER and coworkers ${ }^{(12-14)}$ measured the dissociation and excitation energies of $\mathrm{CaO}, \mathrm{SrO}$ and $\mathrm{BaO}$ in $\mathrm{CO}$-flames. The dissociation energy values derived by the second- and third-law methods could only be reconciled by assuming an electronic partition function $Q_{\mathrm{et}}=6$ (which they tentatively interpreted as a ${ }^{3} \Pi$ ground state). NEwBURY and coworkers ${ }^{(15)}$ made mass-spectrometric and vacuum-thermobalance studies of barium oxide between 1365 and $1917 \mathrm{~K}$ and then calculated the enthalpy for the reaction $\mathrm{BaO}$ (solid) $\rightarrow \mathrm{BaO}$ (gas) by using second- and third-law methods. It appeared from their calculations that the third-law values were only consistent with their second-law values if an electronic partition function of at least $Q_{\mathrm{el}}=6$ was assumed. More recently, OTTINGER and $\mathrm{ZARE}^{(16)}$ studied the chemiluminescence spectrum of some group IIa metal oxides in a crossed-beam experiment. For the $\mathrm{Ba}+\mathrm{NO}_{2}$ reaction, the chemiluminescence was assigned to the $\mathrm{BaO}\left(A^{1} \Sigma \rightarrow X^{1} \Sigma\right)$ band system. In their attempt to account for the $\mathrm{Ba}+\mathrm{N}_{2} \mathrm{O}$ results, they assumed the presence of an intermediate triplet state. CARLSON et al. ${ }^{(17)}$ made Hartree-Fock calculations of the electronic structure of $\mathrm{CaO}$. In this approximation, the lowest states, in order of increasing energy, are: ${ }^{3} \Pi,{ }^{1} \Pi,{ }^{3} \Sigma^{+}$and ${ }^{1} \Sigma^{+}$. They used an open-shell configuration for the ${ }^{1} \Sigma^{+}$term and suggested that this state is actually depressed below the ${ }^{3} \Pi$ state by a strong configuration interaction, giving a $X^{\top} \Sigma$ ground state. $\mathrm{BaO}$ has energy levels resembling those of $\mathrm{CaO}$. They concluded that, on the basis of available calculations, there can be no doubt that the ${ }^{3} \Pi$ and ${ }^{3} \Sigma^{+}$levels lie among the low-lying singlets and perhaps quite close to the ${ }^{1} \Sigma^{+}$ground state. FIELD ${ }^{(18)}$ re-analyzed the experimental results of LAGERQVIST and coworkers, ${ }^{(10)}$ with utilization of perturbation matrix elements for the assignment of perturbations in electronic spectra. It proved possible to assign perturbations of the $A^{1} \Sigma^{+}$state to ${ }^{1} \Pi\left(A^{\prime \prime} \Pi\right)$ and ${ }^{3} \Pi$ states $\left(a^{3} \Pi\right)$. He concluded that $X^{1} \Sigma$ is the ground state of the alkaline-earth monoxides.

From this survey of rather contradictory results, it is clear that further information is still needed. In this paper, two experiments are described. Excitation energy differences are derived directly from the ratios of thermal band intensities measured as a function of flame temperature. Absolute excitation energies are derived from the temperature dependence of the ratio of thermal band to barium line intensity on the assumption of a most probable value for the dissociation energy of $\mathrm{BaO}$.

\section{THEORETICAL ANALYSIS}

\section{A. The band-to-band intensity ratio}

For the temperature dependence of the intensity ratio of two rotational lines (indicated by indices 1 and 2) of the same electronic transition but belonging to different vibrational bands, it follows from equation (2) of Ref. (19) in the case of a uniform flame that

$$
\ln \left\{\left(I_{\mathrm{rot}}\right)_{1} /\left(I_{\mathrm{rot}}\right)_{2}\right\}=c_{1}-\left\{\left(E_{v^{\prime}}+E_{r^{\prime}}\right)_{1}-\left(E_{v^{\prime}}+E_{r^{\prime}}\right)_{2}\right\} / k T,
$$

where $E_{v^{\prime}}+E_{r^{\prime}}=$ the sum of vibrational and rotational energies in the upper state and $c_{1}$ includes all factors which are temperature independent. The semi-logarithmical plot of the intensity ratio vs $1 / T$ yields directly the difference of excitation energies for the two rotational lines considered. 


\section{B. The band-to-line intensity ratio}

The temperature dependence of the intensity ratio for a specific rotational line of a monoxide $M O$ and an atomic line of the same element, $M$, in the absence of self-absorption and for a uniform flame, follows from equations (1) and (2) of Ref. (19), viz.

$$
I_{\text {rot }} / I_{\text {at.line }}=c_{2}\left(n_{M O} / n_{M}\right)\left(Q_{M} / Q_{M O}\right) \exp \left[-\left(E_{u}+E_{v^{\prime}}+E_{r^{\prime}}-E_{\text {at.line }}\right) / k T\right]
$$

where $n_{M}, n_{M O}=$ the number density of atoms $M$ and molecules $M O$, respectively, $Q_{x}=$ the internal partition function of species $X, E_{u}+E_{v^{\prime}}+E_{r^{\prime}}=$ the sum of electronic, vibrational and rotational energies in the upper state of the molecular transition, and $E_{\text {at.line }}=$ the atomic excitation energy. The density ratio $n_{M o} / n_{M}$ can be derived from the equilibrium constant $K_{1}^{\prime}$ for the formal dissociation equilibrium $M O \rightleftarrows M+O$ through the mass-action-law

$$
n_{M O} / n_{M}=p_{M O} / p_{M}=p_{o} / K_{1}^{\prime}
$$

where $p$ denotes the partial pressures at equilibrium. [Even when the actual 0-pressure in the flame deviates from thermal equilibrium, the actual ratio $n_{M o} / n_{M}$ still conforms to equilibrium and is given by equation (3) if we insert therein the calculated equilibrium value for $p_{0}$. This conclusion holds because the dominant formation (and dissociation) reactions for $M O$ do not involve free $O$-atoms as partners (see Ref. 4)]. The constant $K_{1}^{\prime}$ can be written as ${ }^{(21)}$

$$
K_{1}^{\prime}=\left(\mu_{M O}\right)^{3 / 2}\left(2 \pi / h^{2}\right)^{3 / 2}(k T)^{S / 2}\left(Q_{M} Q_{\mathrm{O}} / Q_{M O}\right) \exp \left[-D_{0}(M O) / k T\right),
$$

with $\mu_{M O}=$ reduced mass of $M O, D_{0}=$ dissociation energy, and $Q_{0} \simeq\left(g_{0}\right)_{o}=$ the statistical weight of the ground state, since the oxygen atom has no low-lying electronic states. Substituting equations (3) and (4) in equation (2) yields

$$
\ln \left\{\left(I_{\text {rol }} / I_{\text {at.line }}\right)\left(T^{5 / 2} p_{0}\right)\right\}=c_{3}-\left\{E_{u}+E_{v^{\prime}}+E_{r^{\prime}}-E_{\text {at.line }}-D_{0}(M O)\right\} / \mathrm{kT}
$$

where $Q_{M o}$ has been eliminated. A plot of the left-hand side against the reciprocal temperature yields the excitation energy, $E_{u}+E_{v^{\prime}}+E_{r^{\prime}}$, from the slope of the straight line obtained, if all other energy values are known.

\section{EXPERIMENTAL CONDITIONS AND FLAME CHARACTERISTICS}

The experimental set up and flame equipment have been described for our previous experiments. ${ }^{(4,19)}$ Table 1 shows the main characteristics of the flames used. The mantle flame had the same composition as the central flame, apart from possible entrainment of surrounding air. In some flames, measurements were performed at the two heights indicated by $a$ and $b$. The concentrations of metal vapours formed in the central flame was low enough to prevent self-absorption; cesium chloride was supplied at a concentration sufficiently high to suppress ionization effects. ${ }^{(14)}$

\section{RESULTS}

First the visible emission bands of $\mathrm{BaO}$ were scanned carefully in the selected set of fiames. Four regions about $20 \AA$ wide were chosen for the band intensity measurements, viz. at 5570 , 5945,6545 , and $6860 \AA$, belonging to the $(3,0),(2,1),(0,2)$ and $(0,3)$ vibrational transitions of the 
Table 1. Flow rates of unburnt gas mixtures and water vapour, heights of measurement, and temperatures for the flames used

\begin{tabular}{|c|c|c|c|c|c|c|c|c|c|}
\hline Flame & $\begin{array}{c}\mathrm{H}_{2} \\
(\mathrm{l} / \mathrm{min})\end{array}$ & $\begin{array}{c}\mathrm{C}_{2} \mathrm{H}_{2} \\
(1 / \mathrm{min})\end{array}$ & $\begin{array}{c}\mathrm{O}_{2} \\
(\mathrm{l} / \mathrm{min})\end{array}$ & $\begin{array}{c}\mathrm{N}_{2} \mathrm{O} \\
(\mathrm{l} / \mathrm{min})\end{array}$ & $\begin{array}{c}\mathrm{Ar} \\
(1 / \mathrm{min})\end{array}$ & $\begin{array}{c}\mathrm{N}_{2} \\
(\mathrm{l} / \mathrm{min})\end{array}$ & $\underset{(\mathrm{mg} / \mathrm{min})}{\mathrm{H}_{2} \mathrm{O}}$ & $\begin{array}{l}\text { Height of } \\
\text { measurement } \\
\text { above burner } \\
\text { (cm) }\end{array}$ & $\begin{array}{l}\text { Measured } \\
\text { temperature of } \\
\text { the central flame } \\
\text { (K) }\end{array}$ \\
\hline 1 & 1.4 & - & 0.7 & - & $5 \cdot 0$ & - & 50 & 1.3 & 1911 \\
\hline \multirow{6}{*}{$\left.\begin{array}{r}2 \\
3 a \\
b\end{array}\right\}$} & 1.8 & - & 0.9 & - & 5.0 & - & 50 & $1 \cdot 3$ & 2067 \\
\hline & \multirow{2}{*}{$4 \cdot 0$} & \multirow[t]{2}{*}{-} & \multirow{2}{*}{$2 \cdot 1$} & \multirow{2}{*}{-} & \multirow{2}{*}{-} & \multirow{2}{*}{7.9} & \multirow{2}{*}{180} & $\{6$ & 2240 \\
\hline & & & & & & & & 3 & 2292 \\
\hline & \multirow[t]{2}{*}{-} & \multirow[t]{2}{*}{$1 \cdot 2$} & \multirow{2}{*}{$2 \cdot 1$} & \multirow[t]{2}{*}{-} & \multirow{2}{*}{-} & \multirow{2}{*}{7.9} & \multirow{2}{*}{180} & $\left\{\begin{array}{l}7 \\
\end{array}\right.$ & 2427 \\
\hline & & & & & & & & 4 & 2461 \\
\hline & $5 \cdot 5$ & - & - & $5 \cdot 5$ & - & - & 108 & $\left\{\begin{array}{l}6 \\
7\end{array}\right.$ & 2848 \\
\hline
\end{tabular}

${ }^{1} \Sigma \leftrightarrows^{1} \Sigma$ system, respectively. ${ }^{(10,22,23)}$ In these spectral regions, overlap of other vibrational transitions $\left(v^{\prime}, v^{\prime \prime}\right)$ than those mentioned above was supposed to be negligible, since possible overlapping vibrational transitions have a lower Franck-Condon factor ${ }^{(24)}$ and/or a higher value of $v^{\prime}$ (and thus a lower population of the upper level). With the monochromator band-pass used $(1 \cdot 1 \AA)$, the rotational lines were partially resolved and appeared as individual peaks superposed on a broad continuum (see Fig. 1). Within the spectral regions observed, the pattern of these peaks was the same in all of the flames used, but the height of the underlying continuum did not vary proportionally with the height of the peaks superposed on it. It is not certain whether this

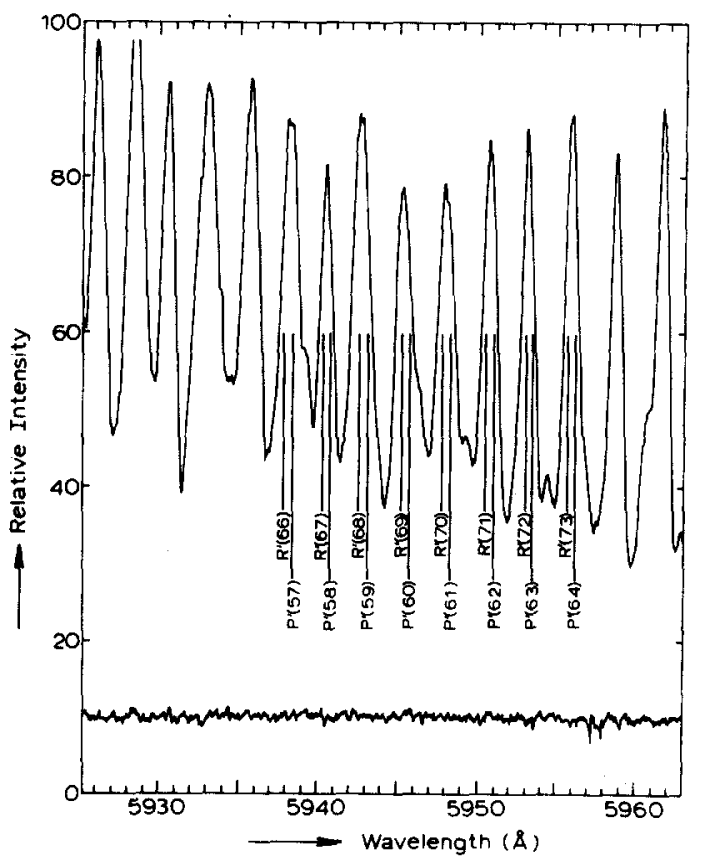

Fig. 1. Part of the $(2,1)$ vibrational emission band of $\mathrm{BaO}$ in the $5945 \AA$ region, as observed in $\mathrm{C}_{2} \mathrm{H}_{2}$-flame no. $4 \mathrm{~b}$ at about $2450 \mathrm{~K}$. The upper spectrogram was obtained while spraying an aqueous $\mathrm{BaCl}_{2}$ solution into the flame; the lower curve is the flame background spectrum. The monchromator band-pass was $0.55 \AA$. The rotational analysis of the peaks at which measurements were done is based on data from Ref. (10). 
(quasi-)continuum arises from other unresolved $\mathrm{BaO}$ or from $\mathrm{BaOH}$ bands. We measured only the height of the peaks with respect to a base line drawn through the minima between the peaks. The wavelengths of the peaks at which measurements were made and the corresponding rotational transitions according to Ref. (10) are listed in Table 2. When a peak is built up from two or more overlapping rotational lines, it is indicated by $R\left(J_{1}\right)+P\left(J_{2}\right)+\ldots$.

We checked the method by measuring the intensity ratios of the rotational lines in the regions at 5570,6545 and $6860 \AA$ to those at $5945 \AA$ four times in all of the flames. The slope of the expected straight line representing $\ln \left\{\left(I_{\text {rot }}\right)_{1} /\left(I_{\text {rot }}\right)_{2}\right\}$ as a function of $1 / T$ [see equation (1)] was determined by means of the least-squares-method; the experimental values were weighted according to their statistical errors. From this slope, the difference in excitation energies of the transitions considered was derived through equation (1). In Table 3, the results of these measurements are compared with the energy differences calculated for the corresponding

Table 2. Peaks of $\mathrm{BaO}$ (in $\AA$ ) observed in the spectral regions studied and corresponding transitions of the ' $\Sigma \rightarrow{ }^{\prime} \Sigma$ band system according to Ref. (10). Overlapping transitions are indicated by $R\left(J_{1}\right)+P\left(J_{2}\right)+\ldots$

\begin{tabular}{|c|c|c|c|}
\hline \multicolumn{2}{|r|}{$\left(v^{\prime}, v^{\prime \prime}\right)=(3,0)$} & \multicolumn{2}{|c|}{$\left(v^{\prime}, v^{\prime \prime}\right)=(2,1)$} \\
\hline$\lambda$ & Rotational lines & $\lambda$ & Rotational lines \\
\hline 5562.9 & $R^{\prime}(66)+P(63)+P^{\prime}(58)$ & 5938.0 & $\mathbf{R}^{\prime}(66)+\mathbf{P}^{\prime}(57)$ \\
\hline 5565.0 & $P^{\prime}(59)$ & $5940 \cdot 5$ & $R^{\prime}(67)+P^{\prime}(58)$ \\
\hline $5569 \cdot 1$ & $R(71)+R^{\prime}(68)$ & 5942.9 & $R^{\prime}(68)+P^{\prime}(59)$ \\
\hline 5571.8 & $R(72)+R^{\prime}(69)+P^{\prime}(62)$ & $5945 \cdot 5$ & $R^{\prime}(69)+P^{\prime}(60)$ \\
\hline 5574.5 & $R(73)+R^{\prime}(70)+P^{\prime}(63)$ & $5948 \cdot 0$ & $R^{\prime}(70)+P^{\prime}(61)$ \\
\hline $5577 \cdot 3$ & $R(74)+P(67)+P^{\prime}(64)$ & 5950.6 & $\mathbf{R}^{\prime}(71)+\mathbf{P}^{\prime}(62)$ \\
\hline \multirow[t]{2}{*}{$5580 \cdot 0$} & $R(75)+P(68)+P^{\prime}(65)$ & 5953.3 & $R^{\prime}(72)+P^{\prime}(63)$ \\
\hline & & 5956.0 & $R^{\prime}(73)+P^{\prime}(64)$ \\
\hline \multicolumn{2}{|r|}{$\left(v^{\prime}, v^{\prime \prime}\right)=(0,2)$} & \multicolumn{2}{|c|}{$\left(v^{\prime}, v^{\prime \prime}\right)=(0,3)$} \\
\hline$\lambda$ & Rotational lines & $\lambda$ & Rotational lines \\
\hline 6536.2 & $R(49)+P(39)$ & $6854 \cdot 3$ & $R(60)+P(50)$ \\
\hline $6540 \cdot 2$ & $R(51)+P(41)$ & 6856.9 & $\mathbf{R}(61)+\mathrm{P}(51)$ \\
\hline $6542 \cdot 3$ & $R(52)+P(42)$ & 6859.6 & $R(62)+P(52)$ \\
\hline $6544 \cdot 4$ & $R(53)+P(43)$ & $6862 \cdot 3$ & $R(63)+P(53)$ \\
\hline \multirow[t]{3}{*}{$6551 \cdot 0$} & $\mathrm{R}(56)+\mathrm{P}(46)$ & $6865 \cdot 0$ & $R(64)+P(54)$ \\
\hline & & 6867.9 & $R(65)+P(55)$ \\
\hline & & $6870 \cdot 8$ & $R(66)+P(56)$ \\
\hline
\end{tabular}

Table 3. Average excitation energy (differences) of the transitions in the spectral regions investigated, as measured by the band-to-band and bandto-line method and as calculated from the molecular constants and known quantum numbers of the states involved

\begin{tabular}{lcc}
\hline \multicolumn{1}{c}{ Energy } & Measured (eV) & Calculated (eV) \\
\hline$E_{5970}-E_{5945}$ & $+0.11 \pm 0.02$ & $+0.068 \pm 0.001$ \\
$E_{6545}-E_{5945}$ & $-0.20 \pm 0.04$ & $-0.185 \pm 0.001$ \\
$E_{6860}-E_{9945}$ & $-0.18 \pm 0.05$ & $-0.149 \pm 0.001$ \\
\hline$E_{5945}-E_{\text {at.tine }}-D_{\mathrm{u}}(\mathrm{BaO})$ & $-4.95 \pm 0.25$ & - \\
\hline
\end{tabular}


transitions specified in Table 2, while using known molecular constants and quantum numbers for the states involved. As can be seen from Table 2, the peaks at which measurements were made, are mostly built up from two or more overlapping rotational lines belonging to different branches. The difference in excitation energy between these branches, $\Delta E$, amounts to about $0.04 \mathrm{eV}$, which gives a variation of 6 per cent in $\exp (-\Delta E / k T)$ when the temperature increases from 1907 to $2886 \mathrm{~K}$. The variation of the excitation energy within each branch, over a spectral range of $20 \AA$, amounts to about $0.02 \mathrm{eV}$, giving a variation of only 3 per cent in the exponential factor. Therefore, we used for $I_{\text {rot }}$ the average value of all peak heights within each $20 \AA$ region. The same averaging procedure was followed in the calculation of the energy differences for the corresponding transitions from known molecular constants. The intensity of all transitions was calculated separately and then averaged over the $20 \AA$ spectral region. The resulting energy difference, also derived from equation (1), is thus a weighted average value which is directly comparable with the measured values. The error limits of the experimental values listed in Table 3 correspond to the maximal and minimal slopes of the lines that can be drawn through the measured points with their statistical errors. The error limits of the calculated values are probably the result of small inaccuracies in the calculations of the intensities for the transitions considered.

The absolute averaged excitation energy of the transitions around $5945 \AA$ was derived from the intensity ratio of these transitions and the atomic Ba-line at $5535 \AA$ by applying equation (5) (see Fig. 2). The result obtained is also listed in Table 3.

\section{DISCUSSION AND CONCLUSIONS}

As discussed in Section 6 of Ref. (20), a correction has to be applied for the underpopulation of the excited atomic level in argon-diluted flames in the absence of self-absorption. The relative correction equals the quantum efficiency of fluorescence $(Y)$, which is known to be relatively large in argon-diluted flames. Since quenching cross sections of the barium resonance line for the major flame constituents were not known, the $Y$-values of the strontium line ${ }^{(25)}$ were used as a rough indication for the importance of this correction. For the two argon-diluted flames used, the correction is estimated to be of the order of 10 per cent.

Chemiluminescence in the cooler flames was supposed to be negligible, since we made measurements far downstream of the flame (i.e. at large heights above the combustion zone) and chemiluminescence is known to decay with increasing height. ${ }^{(26)}$ Moreover, in flames for which measurements were made at two different heights (see Table 1), the corresponding experimental points lie reasonably well on the same straight line drawn through all of the data points (see Fig. 2).

As was mentioned in Section 4, we took the height of the peaks with respect to a base line drawn through the minima between the peaks in the spectrogram as a measure of the intensity of the rotational lines. These heights were averaged over all peaks within each $20 \AA$ region. The choice of base line is rather arbitrary. In order to check whether the intensity ratios $\left(I_{\text {rot }}\right)_{1} /\left(I_{\text {rot }}\right)_{2}$ and $I_{\text {rot }} / I_{\text {line }}$ depend on the choice of base line, we also drew a base line through the maxima and measured the depths of the minima in the $5945 \AA$ region in three flames at 2067,2292 and $2886 \mathrm{~K}$. It appeared that, within an error of 2 per cent, there was no difference in the results obtained with the two base lines. Let us assume the presence of a number of partially overlapping lines with a triangular line profile superposed on a continuum background that varies linearly with the wavelength. A base line is drawn through the minima between the peaks. In this case, it can be proved that the ratios of the heights referred to the base line vary proportionally with the superposed structure, independently of the slope or shape of the continuous background spectrum. 


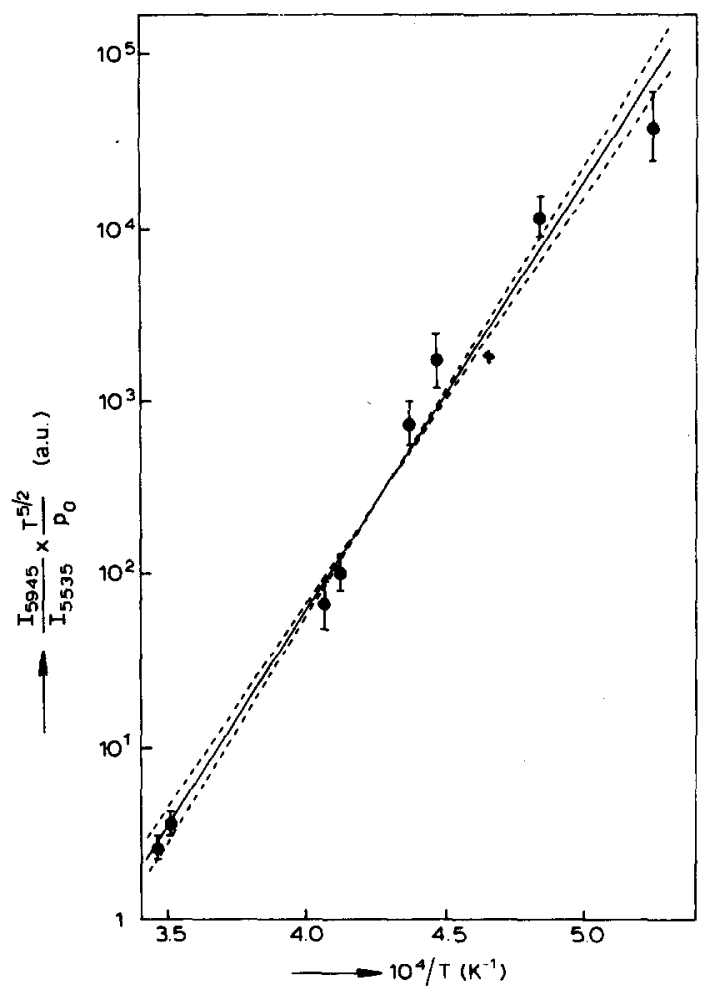

Fig. 2. Semi-logarithmical plot of the band-to-line intensity ratio of $\mathrm{BaO}$, modified according to equation (5), vs $1 / \mathrm{T}$ for determination of the average absolute band excitation energy of the transitions in the $5945 \AA$ region. Variances in reproducibility are indicated by vertical bars. The slope of the straight line indicated was determined by means of the least-squares method. The maximum and minimum acceptable slopes are indicated by dashed lines.

As can be seen from Table 3, the average excitation energy differences for the transitions in the region at 6545 and $6860 \AA$ with respect to those at $5945 \AA$ agree with the calculated values within experimental error. This is not the case for the transitions at $5570 \AA$, probably because of strong perturbation of the upper rotational levels for $v^{\prime}=3$ and $J^{\prime}=60$ to $J^{\prime}=80$ [see Ref. (10) pp. 1145 and 1148], which was not taken into account in the calculated energy differences.

We used the value $D_{0}(\mathrm{BaO})=5 \cdot 30 \pm 0 \cdot 10 \mathrm{eV}$ for the dissociation energy of $\mathrm{BaO}$, as recommended by KALFF, ${ }^{(27)}$ this being a weighted average of his results, RYABOVA and GURVICH'S ${ }^{(28)}$ results which were obtained independently of band excitation energies, and those of SCHOFIELD. ${ }^{(29)}$ Kalff's value was obtained for an assumed electronic partition function $Q_{\mathrm{el}}=6$ for the molecule. The $D_{0}$-values corresponding to other assumed values of $Q_{\mathrm{e} 1}$ are listed in Table 4 .

From the measured band-to-line ratio, the atomic excitation energy $E_{\text {at.line }}=2.24 \mathrm{eV}$, and the various possible values of the dissociation energy (see Table 4), one obtains average values for the absolute excitation energy, $E_{u}+E_{v^{\prime}}+\bar{E}_{r^{\prime}}$ of the rotational transitions in the $5945 \AA$ region, as listed in Table 4. When we assume that the lower electronic state involved in the transitions observed $\left({ }^{1} \Sigma\right)$ is the electronic ground state, the average value of the absolute excitation energy in the $5945 \AA$ region can also be calculated from the molecular constants and the quantum numbers of the states involved. As mentioned above in connection with the calculation of energy 
differences, we then obtain a weighted average value $E_{u}+E_{v^{\prime}}+\overline{E_{r^{\prime}}}=2 \cdot 40 \pm 0 \cdot 01 \mathrm{eV}$. Comparing this value with the experimental values listed in Table 4 for various assumed $Q_{e 1}$ values, we conclude that only the assumption $Q_{\mathrm{e} 1} \simeq 6$ is consistent with the assumption of a ground state transition. Therefore, there must be low-lying electronic states near the ground state giving an electronic partition function of at least $Q_{\mathrm{el}} \simeq 6$.

When the assumption of a ground-state transition is dropped, one finds that the lower ${ }^{1} \Sigma$ state involved in the optical transition lies not more than $0 \cdot 8 \mathrm{eV}$ above the ground state. This maximum value applies if we assume that this state is the only low-lying excited state while the ground state is a ${ }^{1} \Sigma$ state, giving $Q_{e 1} \simeq 1 \cdot 1$ at $T=2886 \mathrm{~K}$. It should be noted that the total internal partition function $Q_{\mathrm{BaO}}$ (and hence also its temperature dependence) cancel in the derivation of equation (5). The electronic partition function is only introduced implicitly because the value of $D_{0}(\mathrm{BaO})$, determined by the third-law method in flames, depends on it.

As a check on the consistency of our measurements, we have also investigated in all flames the temperature dependence of the intensity ratios of the $\mathrm{BaO}$ transitions in the $5945 \AA$ region with respect to the sodium D-line at $5890 \AA$ (the sodium-comparison-method)..$^{(13,14)}$ This method of measuring excitation energies has the advantage that it involves only the fraction of total $\mathrm{Ba}$-concentration that is present in the flame as monoxide, which varies little in flames where $\mathrm{BaO}$ is the dominant species. In our lower-temperature flames, however, $\mathrm{BaO}$ is not dominant and, therefore, besides the dissociation energy $D_{0}(\mathrm{BaO})$, values for $D_{0}(\mathrm{BaOH})$ and $D_{0}\left[\mathrm{Ba}(\mathrm{OH})_{2}\right]$ and a measurement of $p_{\mathrm{H}} / p_{\mathrm{H}_{2} \mathrm{O}}$ through the $\mathrm{Li} / \mathrm{LiOH}$-method ${ }^{(4.19)}$ are required. Using the dissociation energies $D_{0}(\mathrm{BaO}), D_{0}(\mathrm{BaOH})$ and $D_{0}\left[\mathrm{Ba}(\mathrm{OH})_{2}\right]$ recommended by $\mathrm{K}_{\mathrm{ALFF}}{ }^{(27)}$ and the dissociation energies of $\mathrm{LiOH}$ and $\mathrm{NaOH}$ from Ref. (19) for a bent model (which was assumed by Kalff), we obtained an average value of the absolute excitation energy of the transitions in the $5945 \AA$ region $E_{u}+E_{v^{\prime}}+\overline{E_{r^{\prime}}}=2.58 \pm 0.25 \mathrm{eV}$, again assuming $Q_{\mathrm{el}}=6$ for $\mathrm{BaO}$. The indicated error refers only to the reproducibility of the measurements and does not include the systematic errors caused by uncertainties in the values of the dissociation energies used. Comparison of the value of excitation energy obtained by the latter method $(2 \cdot 58 \pm 0.25 \mathrm{eV})$ and the value obtained by the former band-to-line method $(2 \cdot 59 \pm 0 \cdot 27)$ shows satisfactory agreement. Moreover, this agreement supports the values of the dissociation energy for $\mathrm{BaOH}$ and $\mathrm{Ba}(\mathrm{OH})_{2}$ recommended by KALFF, since in the band-to-line method only $D_{0}(\mathrm{BaO})$ is involved, whereas in the sodiumcomparison-method also $D_{0}(\mathrm{BaOH})$ and $D_{0}\left[\mathrm{Ba}(\mathrm{OH})_{2}\right]$ are involved. Nevertheless, we did not use the sodium comparison-method as a basis for our measurements because the dominance of $\mathrm{BaOH}$ and $\mathrm{Ba}(\mathrm{OH})_{2}$ in the lower temperature flames may introduce unknown systematic errors due to uncertainties in the corresponding dissociation energies. The band-to-line method has the advantage that any shift in the adopted $D_{0}(\mathrm{BaO})$ value yields an equally large shift in the excitation energy value [see equation (5)].

Table 4. Recommended dissociation energies of $\mathrm{BaO}$ [see Ref. (27)] for various assumed values of the electronic partition function, $Q_{\mathrm{el}}$, and corresponding average excitation energies of the transitions in the $5945 \AA$ region, as obtained from band-to-line measurements (see also Table 3 )

\begin{tabular}{ccc}
\hline Assumed $Q_{\mathrm{el}}$ & $D_{\mathrm{o}}(\mathrm{BaO})(\mathrm{eV})$ & $E_{\mathrm{u}}+\dot{E}_{v}+\bar{E}_{\mathrm{r}}(\mathrm{eV})$ \\
\hline 6 & $5 \cdot 30 \pm 0 \cdot 10$ & $2 \cdot 59 \pm 0 \cdot 27$ \\
3 & $5 \cdot 45 \pm 0 \cdot 10$ & $2 \cdot 74 \pm 0.27$ \\
1 & $5 \cdot 69 \pm 0 \cdot 10$ & $2 \cdot 98 \pm 0.27$ \\
\hline
\end{tabular}


In conclusion, it may be stated that the vibrational and rotational term values involved in the transitions considered, as listed in the spectroscopic handbooks, ${ }^{(22.23)}$ are confirmed by our measurements. If the lower electronic state involved in these transitions $\left({ }^{1} \Sigma\right)$ is the ground state, other low-lying electronic states must be present in order to make $Q_{e l}=6$. It is, however, unclear why no optical transitions terminating at these additional states have been observed in the barium spectrum.

\section{REFERENCES}

1. A. G. GaYDon, Dissociation Fnergies. Chapman \& Hall, London (1968).

2. A. LAGerQvist and L. HuldT, Ark. Fys. 8, 427 (1954).

3. L. HuldT and A. LAGERQvist, Ark. Fys. 9, 227 (1955).

4. J. VAN DER HURK, TJ. HOLLANDER and C. TH. J. AlKEMADE, JQSRT 13, 273 (1973).

5. I. V. VeiTS and L. V. GURVICH, Optika $i$ Spektrosk. 2, 145 (1957).

6. E. M. BuLEWICZ and T. M. SUGDEN, Trans. Faraday Soc. 55, 720 (1959).

7. J. DrowarT, G. EXSTEEN and G. VERHAEGEN, Trans. Faraday Soc. 60, 1920 (1964).

8. L. Wharton, M. KaUfman and W. Klemperer, J. chem. Phys. 37, 621 (1962).

9. L. Wharton and W. KLemperer, J. chem. Phys. 38, 2705 (1963).

10. A. Lagerqvist, E. Lind and R. F. Barrow, Proc. phys. Soc. A63, 1132 (1950).

11. M. Kaufman, L. WharTon and W. Ki.mpperer, J. chem. Phys. 43, 943 (1965).

12. Tj. Hollander, P. J. KalfF and C. Th. J. Alkemade, JQSRT 4, 577 (1964).

13. P. J. KalfF, Tj. Hollander and C. Th. J. Alkemade, J. chem. Phys. 43, 2299 (1965).

14. Tj. HollandER, Ph.D. Thesis, Utrecht (1964).

15. R. S. Newbury, G. W. Barton and A. W. Searcy, J. chem. Phys. 48, 793 (1968).

16. Ch. Ottinger and R. N. Zare, Chem. phys. Lett. 5, 243 (1970); C. D. Jonah, R. N. ZARE and Ch. OtTinger, J. chem. Phys. 56, 263 (1972).

17. K. D. Carlson, K. Kaiser, C. Moser and A. C. WaHL, J. chem. Phys. 52, 4678 (1970).

18. R. W. Field, J. chem. Phys. 60, 2400 (1974).

19. J. van der HuRk, TJ. Hollander and C. TH. J. Alkemade, JQSRT 14, 1167.

20. E. M. Bulewcz, C. G. James and T. M. Sugden, Proc. R. Soc. A235, 89 (1956).

21. R. FowLER and E. A. GUGgenheIm, Statistical Thermodynamics. University Press, Cambridge (1965).

22. R. Mavrodineanu and H. Borteux, Flame Spectroscopy, Wiley, New York (1965).

23. B. Rosen, Données Spectroscopiques Relatives aux Molécules Diatomiques. Pergamon Press, Oxford (1970).

24. T. WENTINK and R. J. SPINDLER, JQSRT 12. 129 (1972).

25. Tj. Hollander, P. L. Lijnse, L. P. L. Franken, B. J. Jansen and P. J. Th. ZeEgers, JPSRT 12, 1067 (1972); TJ. Hollander, P. L. LiNSE, B. J. JANSEN and L. P. L. Franken, JQSRT 13, 669 (1973).

26. T. M. Sugden, Annual Review of Physical Chemistry (Edited H. Eyring), p 369. Annual Reviews Palo Alto. (1962).

27. P. J. KalfF, Ph.D. Thesis, Utrecht (1971); P. J. KalfF and C. Th. J. Alkemade, J. chem. Phys. 59, 2572 (1973); P. J. KaLFF and C. Th J. Alkemade, Erratum, J. chem. Phys. 60, 1698 (1974).

28. V. G. Ryabova and L. V. GuRvich. High Temp. 3, 284 (1965).

29. K. SCHOFIELD, Chem. Rev. 67, 707 (1967). 